\title{
Access Pricing Under Imperfect Competition Reconsidered $^{1}$
}

\section{Rostislav Staněk ${ }^{2}$ and Michal Kvasnička ${ }^{3}$}

\begin{abstract}
This paper claims that Onemli's results published in "Access Pricing under Imperfect Competition", Review of Economic Perspectives, 2012, are incorrect. Contrary to Onemli, we claim that in an industry, where a monopoly incumbent produces a key input used by itself and its competitors on a downstream market which is Cournot oligopoly, the regulator should set the second-best access charge such that the incumbent's total profit is zero if the first-best access charge is not feasible. The competitors' ability to produce the key input themselves does not change the outcome since no competitor chooses to use this option under this regulation. We also discuss some limitations of the Onemli's model.
\end{abstract}

Key words: regulation, second-best optimal access charge, monopoly, Cournot oligopoly

JEL Classification: L19, L51, L97

\section{Introduction}

In this paper, we revise the results that Onemli published in his paper "Access Pricing Under Imperfect Competition", Review of Economic Perspectives, 2012. Following Spencer and Brander (1983) and Vickers (1995), Onemli studies how to regulate "industries that contain both naturally monopolistic and potentially competitive activities" (Vickers, 1995, p. 1). Examples of such industries include telecommunications, electric power industry, natural gas industry, railways and others. Onemli (2012) considers an industry that consists of a vertically integrated firm (the incumbent) and its competitors. The incumbent operates on two subsequent markets. On the upstream market, the incumbent is a monopoly producer of an intermediate product. On the downstream market, it competes with the other firms in production of a final good. All firms on the downstream market (including the incumbent) use the intermediate product sold on the upstream market as the key input. An example of such a structure may exist in the railroads industry. Suppose that in the past a vertically integrated monopoly operated both the tracks (the upstream market) and the passenger

\footnotetext{
${ }^{1}$ This paper has been created as a part of project of specific research no. MUNI/A/0796/2011 at the Masaryk University.

${ }^{2}$ Masaryk University, Economic Faculty, Lipová 41a, Brno, 602 00, the Czech Republic, rostanek@mail.muni.cz

${ }^{3}$ Masaryk University, Economic Faculty, Lipová 41a, Brno, 602 00, the Czech Republic, michal.kvasnicka@econ.muni.cz
} 
and freight service (the downstream market). Later, the monopoly incumbent was forced to allow competition in the passenger and freight service while retaining the ownership of the tracks. The competitors have to pay access charge for the use of the tracks (the key input produced by the incumbent) which is usually set by a regulator.

The organization and regulation of such industries poses many interesting questions. For instance, Vickers (1995) asked whether the incumbent should be allowed to operate on the downstream market, Spencer and Brander (1983) asked what the optimal level of theaccess chargeis when the incumbent's profit must not be lesser than a given amount. Onemli (2012) asked a question similar to the question raised by Spencer and Brander (1983). First he shows that the first-best level of the access charge is lower than incumbent's marginal cost, and hence the incumbent's corresponding profit is negative. To make the incumbent produce at all, the regulator has to set the second-best level of the access charge such that the incumbent's profit or its part is non-negative (the participation constraint). Then he asks whether it is optimal to impose the participation constraint on the incumbent's upstream activities only, or on its overall profit. He explicitly assumes a linear demand curve.

Onemli's major results are summarized in his Proposition 2. They are as follows:

1. The welfare is higher when the participation constraint is imposed only on the incumbent's upstream activities.

2. When the participation constraint is imposed only on the incumbent's upstream activities the incumbent's profit is lower than when the constraint is imposed to the incumbent's overall profit.

These conclusions are wrong. We will deal with the Onemli's model and show that his conclusions hold true the other way round for a large class of demand functions, including the linear one he used. Onemli came to wrong conclusions because he solved his model incorrectly. Because his Proposition 2 is incorrect, we have to providea new analysis of the bypass problem (his Proposition 3) too. We will show that his Proposition 3 is incorrect, too. Up to this point, we stick with the Onemli's model formulation (though we generalize on the demand function form). At the end of this paper, we discuss other limitations of the (correctly solved) Onemli's model.

\section{Model}

In conformity with Onemli (2012), we assume an industry that consists of two markets (the upstream and downstream ones) and two kinds of firms (one incumbent and its $n-1$ competitors). The incumbent is a vertically integrated firm that operates on both these markets. In the upstream market, the incumbent is a monopoly producer of an intermediate product. The intermediate goodis the only and essential input for production of the final good produced on the downstream market. Each unit of the final product is made from one unit of the intermediate good. The incumbent sells the intermediate good to its competitors on the downstream market for the access charge $w$. The downstream market is characterized by Cournot oligopoly. On the downstream 
market, the incumbent competes with its $n-1$ competitors. The downstream product is homogenous and is sold to the final customers at a price $p$. The final price $p$ is given by an inverse demand function $p=P(Q)$, where $Q=q_{1}+q_{2}+\cdots+q_{n}$ is the total industry production, i.e. the sum of quantities produced by the incumbent $q_{1}$ and its competitors $q_{j}, j=2, \ldots, n$. Only the production of the intermediate product is costly; its transformation to the final good has a zero cost.

The incumbent's total cost function is $C_{1}(Q)=c Q$, i.e. its marginal cost is constant $c$ and there is no fixed cost. Its profit $\Pi_{1}$ consist of two parts: the upstream profit $\Pi_{U}$, which is the margin charged to its competitors $(w-c)$ times the amount of inputs they buy (which is equal to the amount of their production), and the downstream profit $\Pi_{D}$ from its own production of final goods, which is their own margin $(p-c)$ times the amount of their production $q_{1}$, i.e.

$$
\Pi_{1}=\Pi_{U}+\Pi_{D}=(w-c) \sum_{j=2}^{n} q_{j}+(P(Q)-c) q_{1} .
$$

Each of its competitors (denoted with the lower index -1) has zero cost of input transformation, hence their cost function is $C_{-1}\left(q_{-1}\right)=w q_{-1}$, i.e. their marginal costsare equal to the access charge $w$ and there is no fixed cost. The competitor's profit is then

$$
\Pi_{-1}=(P(Q)-w) q_{-1} .
$$

Assumption 1: We assume that the inverse demand function $P(Q)$ is decreasing and satisfies the following property: $P^{\prime}(Q)+q_{i} P^{\prime \prime}(Q)<0$ for each $q_{i}$. This property states that the marginal revenue is decreasing. It is a sufficient condition that ensures that an equilibrium of Cournot model does exist, see Tirole (1988, p. 219 and 224-228) or Vives (1999, p. 94-96). Note that the linear inverse demand curve $P(Q)=a-b Q$ used by Onemli (2012)is a special case. Next we assume $P^{-1}(c)>0$, i.e. there exists an equilibrium where positive quantities are produced. We also assume that the demand function is derived from quasi-linear preferences.

The access charge $w$ can be set by the incumbent (if it is unregulated) or by a regulatory agency which is assumed to by fully-informed. The model then must be solved as a twostage extensive game. In the first stage, the optimal access charge $w$ is set (either by the incumbent to maximize its profit, or by the regulator to maximize the overall welfare). In the second stage, all firmssimultaneouslydecide on how much to produce given the access charge $w$ determined in the previous stage. Thus the model has to be solved by the backward induction.

The Nash equilibrium in the second stageis determined by the intersection of the reaction functions, i.e. given by the solution of the following system of first order conditions: 


$$
\begin{aligned}
\frac{d \Pi_{1}}{d q_{1}} & =P(Q)+P^{\prime}(Q) q_{1}-c=0, \\
\frac{d \Pi_{-1}}{d q_{-1}} & =P(Q)+P^{\prime}(Q) q_{-1}-w=0 .
\end{aligned}
$$

We denote the equilibrium quantity supplied by firm $i$ at a given value of the access charge $w$ as $q_{i}^{*}$ and the market equilibrium quantity supplied at a given value of the access charge $w$ by all firms as $Q^{*}$.

The relationship between the equilibrium price and the value of the access charge is derived in Lemma 1.

Lemma 1: If the competitors produce a positive quantity $q_{-1}>0$ in the downstream market then the equilibrium final price must be greater than the access charge:

$$
P\left(Q^{*}(w)\right)>w .
$$

Proof: We can reformulate the first order condition (3) as $P(Q)=w-P^{\prime}(Q) q_{-1}$. Since $P^{\prime}(Q)<0$, then $P\left(Q^{*}(w)\right)>w$.

Other important properties concerning the equilibrium quantities are summarized in Lemma 2.

Lemma 2: Suppose that Assumption 1 holds and $q_{-1}>0$. If the access charge $w$ increases then the incumbent's quantity $q_{1}^{*}$ increases and the equilibrium market quantity $Q^{*}$ decreases.

Proof: Allowing for the infinitesimal change in the access charge and differentiating the first order conditions (3), we get the following system of $n$ equations

$$
\begin{gathered}
P^{\prime}\left(Q^{*}\right) d q_{1}^{*}+P^{\prime \prime}\left(Q^{*}\right)\left(d q_{1}^{*}+\sum_{j=2}^{n} d q_{j}^{*}\right) q_{1}^{*}+P^{\prime}\left(Q^{*}\right)\left(d q_{1}^{*}+\sum_{j=2}^{n} d q_{j}^{*}\right)=0, \\
P^{\prime}\left(Q^{*}\right) d q_{-1}^{*}+P^{\prime \prime}\left(Q^{*}\right)\left(d q_{1}^{*}+\sum_{j=2}^{n} d q_{j}^{*}\right) q_{-1}^{*}+P^{\prime}(Q *)\left(d q_{1}^{*}+\sum_{j=2}^{n} d q_{j}^{*}\right)=d w .
\end{gathered}
$$

By solving this system of equations, we obtain two formulas which state the change in the incumbent's quantity and change in the competitor's quantity induced by the change of the access charge.

$$
\begin{gathered}
d q_{-1}^{*}=d w \frac{q_{1}^{*} P^{\prime \prime}\left(Q^{*}\right)+2 P^{\prime}\left(Q^{*}\right)}{P^{\prime}\left(Q^{*}\right)\left[P^{\prime \prime}\left(Q^{*}\right)\left((n-1) q_{-1}^{*}+q_{1}^{*}\right)+P^{\prime}\left(Q^{*}\right)(n+1)\right]} \\
d q_{1}^{*}=-d w \frac{(n-1)\left(P^{\prime \prime}\left(Q^{*}\right) q_{1}^{*}+P^{\prime}\left(Q^{*}\right)\right)}{P^{\prime}\left(Q^{*}\right)\left[Q^{*} P^{\prime \prime}\left(Q^{*}\right)+(n+1) P^{\prime}\left(Q^{*}\right)\right]}
\end{gathered}
$$

After considering the downward-sloping marginal revenue condition $P^{\prime}(Q)+$ $q_{i} P^{\prime \prime}(Q)<0$, we can see that $d q_{-1}^{*} / d w<0$ and $d q_{1}^{*} / d w>0$. The change in the 
market quantity $d Q^{*}$ is the sum of the change in incumbent's quantity and the change in the quantities supplied by its $n-1$ competitors.

$$
d Q^{*}=d q_{1}^{*}+(n-1) d q_{-1}^{*}=d w \frac{(n-1) P^{\prime}\left(Q^{*}\right)}{P^{\prime}\left(Q^{*}\right)\left[Q^{*} P^{\prime \prime}\left(Q^{*}\right)+(n+1) P^{\prime}\left(Q^{*}\right)\right]} .
$$

Again, the downward-sloping marginal revenue condition ensures that $d Q^{*} / d w<0$. This completes the proof.

Since we assume quasi-linear preferences, the overall welfare is an unweighted sum of consumers' surplus and the profits of all the firms. It is easy to see that under the assumed cost structure the overall welfare $W$ is

$$
W=\int_{0}^{Q^{*}}(P(x)-c) d x
$$

Taking into account equilibrium quantity on the downstream market, we can show how the overall welfare reacts to changes in the access charge $w$. This result is derived in Lemma 3.

Lemma 3: Overall welfare $W$ increases when access charge $w$ decreases until the point when the final price equals marginal costs, i.e. $P\left(Q^{*}\right)=c$.

Proof: Taking the derivatives of the overall welfare according to the access charge $w$, we obtain

$$
\frac{\partial W}{\partial w}=\left(P\left(Q^{*}\right)-c\right) \frac{\partial Q^{*}}{\partial w} .
$$

We know that $\partial Q^{*} / \partial w<0$, whichmeans that $\partial W / \partial w<0$ as long as $P\left(Q^{*}\right)>c$.

\section{Welfare analysis}

In this section, we compare welfare consequences of various settings of the access charge:1) the access charge set by an unregulated incumbent, 2) the access charge set by the regulator not restricted by the participation constraint, and 3) the access charge set by the regulator subject to the participation constraint. Before presenting a formal analysis, we give a heuristic explanation of our results. The results are summarized in proposition 1 . The formal proof can be found in the appendix.

First, consider the case when the access charge is set by the unregulated incumbent. The incumbent can set the access charge so high that no other firm wants to enter the downstream market, i.e. $q_{-1}=0$. Under this access charge, the incumbent produces the monopoly quantity $q_{1}^{M}=Q^{M}$. The incumbent then obtains the monopoly profit $\Pi^{M}=$ $\left(P\left(Q^{M}\right)-c\right) Q^{M}$. There are many values of the access charge $w^{M}$ that ensure zero production of other competitors; denote the set of such access chargesas $v^{M}=$ $\left\{w: q_{-1}^{*}(w)=0\right\}$. We claim that any $w^{M} \in v^{M}$ is the optimal access charge for a profit maximizing incumbent. In order toprove this claim, note that the sum of profits in the 
industry can never be greater than the monopoly profit, and other competitors will enter the market if and only if their profits are non-negative. Hence, the profit of the incumbent can never be greater than the monopoly profit, if some competitors are active on the downstream market. We denote overall welfare in this case as $W^{M}$.

Second, consider the case when the access charge is set up by the regulator which wants to maximize the overall economic welfare with no respect to the participation constraint. It follows directly from Lemma 3 that the first-best solution which maximizes the overall welfare is to set such an access charge that the final price equals production costs, i.e. $P\left(Q^{*}\right)=c$. By Lemma 1 , the first best access charge $w^{F B}$ is then smaller than the marginal costs, i.e. $w^{F B}<c=P\left(Q^{*}\right)$. The reason is that the downstream market is characterized by Cournot competition which results in a positive mark-up over marginal costs. In this case, the incumbent has negative profit on the upstream market because $w^{F B}<c$, and zero profit on the downstream market because $P\left(Q^{F B}\right)=c$. The incumbent's total profit $\Pi_{1}^{F B}$ is then negative. Equilibrium values in this situation are indexed by superscript FB.

The first best solution requires the regulator to be able to raise funds by non-distortive taxes and make a transfer to the incumbent to coverits loss. If this is not the case, then the first-best solution is not feasible because the incumbent is not willing to produce the intermediate product at all. Therefore, we have to analyze the second-best situation when the regulator is restricted by the incumbent's participation constraint. Following Onemli (2012), we ask whether the regulator should impose the participation constraint on the incumbent's total profit $\Pi_{1}=\Pi_{U}+\Pi_{D}$ or only on the profit from its upstream activities $\Pi_{U}$. The problem faced by the regulator is then

$$
\begin{gathered}
\max _{w, \theta} W=\int_{0}^{Q^{*}}(P(x)-c) d x \\
\text { subject to } \Pi_{U}+\theta \Pi_{D} \geq 0, \text { where } \theta \in\{0,1\} .
\end{gathered}
$$

We solve the problem in two steps. First, we find the optimal access charges $w^{U}$ and $w^{O}$ for $\theta=0$ (the participation constraint applied only to the incumbent's upstream profit) and $\theta=1$ (the constraint applied to its total profit) respectively. Then we compare the corresponding welfare $W^{U}$ to $W^{O}$. The formal analysis is postponed to Appendix but the intuition is straightforward. Lemma 3 shows that the regulator maximizing the welfare wants to set the lowest possible access charge until the participation constraint is binding. The optimal values of the access charge $w^{U}$ and $w^{O}$ are therefore fully determined by participation constraints $\Pi_{U}=0$ and $\Pi_{U}+\Pi_{D}=0$, respectively. To explain the intuition behind the result, consider the situation when $\theta=0$. Obviously, the optimal access charge is equal tothe marginal cost, i.e. $w^{U}=c$. The incumbent's total profit $\Pi_{1}^{U}$ is then positive, because incumbent obtains a positive profit on the downstream market. Hence, there is room for lowering the access charge and increasing the welfare, if the participation constraint is imposed on incumbent's overall profit instead. 
Results of this analysis are summarized in Proposition 1. Besides others, the proposition shows that the incumbent's profit is higher when the participation constraint applies only to its upstream activities. The overall welfare is higher when the participation constraint applies to the incumbent's overall profit. Note that these results are exactly opposite to the results presented in Proposition 2 in Onemli (2012).

Proposition 1: Consider the four cases stated above.By comparison of the incumbent's profit, it holdsthat $\Pi_{1}^{M}>\Pi_{1}^{U}>\Pi_{1}^{O}>\Pi_{1}^{F B}$. By comparison of the overall welfare, it holds that $W^{F B}>W^{O}>W^{U}>W^{M}$.

For the formal proof see Appendix.

\section{Bypass}

Onemli (2012) also explores the possibility of a bypass. He assumes $m$ of $n-1$ competitors can themselves produce the key input at the marginal $\cos t c$, i.e. at the same cost as the incumbent. Our previous analysis allows us to study consequences of the bypass in a simple way that requires no extra computation. Let us explore all four cases considered above informally first; the results are then summarized in Proposition 2.

First, let us consider the case that the incumbent is not regulated. When there is no bypass, the incumbent sets the access price to the level $w^{M} \in v^{m}$ which secures it a monopoly position. If $m$ competitors can bypass, the monopoly changes to a symmetric Cournot model with $m+1$ symmetric vertically integrated firms. Such a change decreases the final price and raises the total quantity, which enhances the overall welfare. Let us denote the resulting overall welfare as $W^{C}$.

Second, let us consider the case that the access price is set by the regulator to its secondbest value $w^{U}$ that maximizes the overall welfare provided that the incumbent's upstream profit is non-negative. As we have seen, $w^{U}=c$. Then the $m$ competitors which can bypass have no incentive to do it-they can buy the input from the incumbent for exactly the same price as is their own cost of producing it. Even if they did bypass, the equilibrium market quantity and price would not change because the firms' cost would be the same as with no bypass. The overall welfare is in this case $W_{B}^{U}=W^{U}$.

Third, let us consider the case that the access price is set by the regulator to its secondbest value $w^{O}$ that maximizes the overall welfare provided that the incumbent's total profit is non-negative. As we have seen, $w^{O}<c$. Then the $m$ competitors which can bypass have no incentive to do it - they can buy the input cheaper from the incumbent for the subsidized access price $w^{O}$ than they can produce it. Since the welfare is decreasing in $w$, the welfare $W_{B}^{O}=W^{O}$ is higher in this case then in the previous one.

Thus the ability to bypass is useless if the access price is set to its second-best value: the downstream competitors have any incentive to bypass whatsoever, only if the incumbent is not regulated or if the access price is set to its first-best value. The welfare comparison is summarized in Proposition 2. 
Proposition 2: It holds that $W^{M}<W^{C} \leq W^{U}=W_{B}^{U}<W^{O}=W_{B}^{O}$, i.e. the bypass improves welfare only if the incumbent is unregulated. In this case, the overall welfare is increasing in the number of bypassing firms $m$. If the regulator sets the access charge to $w^{U}$ or $w^{O}$, the ability to bypass does not change the equilibrium welfare; the equilibrium welfare is then independent of $m$.

Proof: First, we compare $W_{B}^{U}$ and $W_{B}^{O}$. It holds that $d \Pi_{i} / d c_{i}<0$, i.e. firm's profit is decreasing in its own marginal costs (see Dixit 1986, p. 113). Hence, no firm bypasses if $w \leq c$. We have proved that $w^{U}=c$ and $w^{O}<c$, which implies that $W_{B}^{O}=W^{O}$ and $W_{B}^{U}=W^{U}$. By proposition $1, W^{O}>W^{U}$, hence $W_{B}^{O}>W_{B}^{U}$.

Now we compare $W_{B}^{U}, W^{C}$ and $W^{M}$. In all cases there is a symmetric Cournot structure on the downstream market with the constant marginal cost $c$. The total output in Cournot equilibrium is increasing in the number of firms (see Amir and Lambson, 2000). There is only one firm in the monopoly case $\left(W^{M}\right)$. There are $m+1 \leq n$ firms in the bypass case $\left(W^{C}\right)$ and $n$ firms in the second-best regulation of the incumbent's upstream profit $\left(W_{B}^{U}=W^{U}\right)$. We then get the result by applying Lemma 2 .

Onemli (2012) claims in his Proposition 3 that the second-best $w$ is decreasing in $m$ and that the overall welfare is increasing in $m$ if the regulator sets $w$ to its second-best level $w^{U}$ or $w^{O}$. This claim is clearly wrong. All the second-best access charges $w^{U}$ and $w^{O}$, and the overall welfare $W^{U}$ and $W^{O}$, are independent of the number of firms which could potentially bypass because none of these firms will in fact choose to bypass under these access charges.

\section{Model limitations}

So far, we have been working within the (slightly generalized version of the) Onemli's (2012) model. Now we will discuss some limitations of the model as such. In particular, we claim that 1) the model structure makes some implicit assumptions; and 2) the model structure without these assumptions can have quite different properties than the (correctly solved) model presented above. Thus the applicability of the model results is somewhatlimited.

The model structure assumes that the incumbent has a monopoly in the upstream market. This assumption cannot be motivated by fixed costs here, as it is usual in the literature (see e.g. Vickers (1995)), unless the preceding analysis is changed substantially. Under the assumption of huge fixed costs it might happen that 1) the incumbent makes a negative profit if the regulator sets the second best access price $w=c$ because the incumbent's downstream profit may be lower than its fixed cost; 2) the second-best access price subject to the constraint that the incumbent's total profit is non-negative might be higher than its marginal cost, i.e. $w>c$. To motivate the monopoly position, the model presupposes some different barriers to entry, such as sunk costs or patented technology. 
A similar assumption is needed on the downstream market too. The model postulates that the number of the incumbent's competitors isfixed to $n$. However, their number should be rather endogenous in the model. But under the competitors' cost structure (zero marginal cost and no fixed cost), infinity of firms would enter the downstream market, changing it to perfect competition. The regulator's task would be easy in such a case. The natural way how to motivate the Cournot structure on the downstream market is to assume that the downstream firms have some positive fixed cost (e.g. see again Vickers (1995)). However, then these fixed costs must be explicitly assumed when the overall welfare is calculated. The calculation of the second-best access price $w$ is then difficult because $d W / d w$ is no longer continuous. It may happen that a decrease in $w$ will increase the overall welfare because the total quantity $Q^{*}$ increases, or decrease the overall welfare because the increase in the total quantity $Q^{*}$ allows an entry of a new firm and the increase of the consumers' surplus may be lower than the fixed cost of the newly entered firm. Thus the model structure is valid only if there are some barriers to entry to the downstream market other than fixed costs.

Third, the model structure supposes that the first-best solution is not feasible. But in some cases, the regulator can provide the incumbent with a fixed fee. Then the regulator can cover incumbent's loss and the first-best access fee may be a viable approach. Hence, the model implicitly assumes that the regulator cannot make such a transfer to the incumbent. One case when this assumption is valid is when the regulator cannot raise the funds with sufficiently low distortion.

Fourth, the model predicts that it is better to set the second-best access price $w$ such that the incumbent's total profit $\Pi_{1}$ is non-negative rather than only its upstream profit $\Pi_{U}$ is non-negative because the overall welfare is higher in the former case. However, in some cases there might be a reason to set the second-best access price such that only the incumbent's upstream profit is non-negative. For instance, if the incumbent's profit is regulated to zero, the owners of incumbent's inputsor its managers may be tempted to inflate artificially the costs to gain extra return or on-the-job consumption. The owners of the incumbent firm have no incentive to resist it, see Eggertsson (1990, pp. 143-149) for a short review. Regulating only the upstream profit can serve as an incentive mechanism in such a case.

\section{Conclusions}

In is paper, we have revisited the results presented in Onemli (2012). Contrary to him, we show that it is welfare-enhancing to impose the non-negativity constraint on the overall profit of the incumbent instead of on the incumbent's upstream activities only. Moreover, we show that welfare in these situations does not depend on the ability of the incumbent's competitors'to bypass.

However, the model structure, and hence these conclusions have some limitations. They are relevant only in the situation that is characterized by following conditions: First, there are barriers to entry both on the upstream and the downstream markets other than fixed cost. Second, the regulator is not willing or able to pay the incumbent a lump-sum 
payment to cover its loss, e.g. because the regulator is not able to raise the necessary funds without sufficiently low distortion. Moreover, the conclusion does not need to hold if the incumbentcan inflate its cost.

\section{References}

AMIR, R.-LAMBSONV. E. (2000). On the Effects of Entry in Cournot Markets. Review of Economic Studies, 67(2), Pp. 235-254.

DIXIT, A. (1986). Comparative Statics for Oligopoly. International economic review, 27(1), Pp. 107-112.

EGGERTSSON, T. (1990). Economic behavior and institutions, Cambridge: Cambridge University Press.

ONEMLI, M. B. (2012). Access Pricing Under Imperfect Competition", Review of Economic Perspectives. 12(1). Pp. 3-21.

SPENCER, B. J.—BRANDER, J. A. (1983). Second Best Pricing of Publicly Produced Inputs. Journal of Public Economics. 20(1), Pp. 113-119.

TIROLE, J. (1988). Theory of Industrial Organization. Cambridge, Massachusetts: The MIT Press.

VICKERS, J. (1995). Competition and Regulation in Vertically Related Markets. Review of Economic Studies. 62(1). Pp. 1-17.

VIVES, X. (1999). Oligopoly Pricing. Cambridge, Massachusetts: The MIT Press. 


\section{Appendix}

Proof of proposition 1: The proofgoes in three steps.

Step 1: $\Pi^{F B}<\Pi^{O}=0$ and $W^{F B}>W^{O}$

The first best access charge $w^{F B}$ is the solution of the unconstrained regulator's problem $\max _{w} \int_{0}^{Q^{*}}(P(x)-c) d x$. It follows from Lemma 3 that the solution of the problem is $w^{F B}$ such that $P\left(Q^{*}\right)=c$. Lemma 1implies that $w^{F B}<c$. Evaluating the incumbent's profit at the first-best access charge, we obtain incumbent's profit $\Pi_{1}^{F B}=$ $(w-c) \sum_{j=2}^{n} q_{j}^{*}+\left(P\left(Q^{*}\right)-c\right) q_{1}^{*}<0$. The second best access charge $w^{O}$ is the solution of the regulator's problem subject to the constraint $\Pi_{1}=0$. It follows from the definition of the problem and the fact that the participation constraint is binding that $w^{O}>w^{F B}$. Lemma 3 then implies second part of the claim.

Step $2: \Pi^{U}>\Pi^{O}=0$ and $\mathrm{W}^{O}>W^{U}$.

Proof: The regulator's problem under participation constraint is

$$
\max _{w} W=\int_{0}^{Q^{*}}(P(x)-c) d x
$$

subject to $\Pi_{U}+\theta \Pi_{D} \geq 0$, where $\theta \in\{0,1\}$.

The solution of the problem is given by Kuhn-Tucker conditions:

$$
\begin{gathered}
\frac{\partial\left[W-\lambda\left(\Pi_{U}+\theta \Pi_{D}\right)\right]}{\partial w}=0, \\
\frac{\partial\left[W-\lambda\left(\Pi_{U}+\theta \Pi_{D}\right)\right]}{\partial \lambda}=\Pi_{U}+\theta \Pi_{D} \leq 0, \\
\lambda \frac{\partial W-\lambda\left(\Pi_{U}+\theta \Pi_{D}\right)}{\partial \lambda}=\lambda\left(\Pi_{U}+\Pi_{D}\right)=0 .
\end{gathered}
$$

Lemma 3 and analysis in the previous step shows that the participation constraint is binding at the optimum in both cases. Kuhn-Tucker conditions are then reduced to the following equations

$$
\begin{gathered}
\frac{\partial W-\lambda\left(\Pi_{U}+\theta \Pi_{D}\right)}{\partial w}=0, \\
\Pi_{U}+\theta \Pi_{D}=0 .
\end{gathered}
$$

The optimal access charge is determined by equation (7), whereas equation (6) determines the value of the Lagrange multiplier. The optimal access charges $w^{U}$ and $w^{O}$ are given by conditions $\Pi_{U}=0$ and $\Pi_{U}+\Pi_{D}=0$ respectively.

The optimal access charge $w^{U}$ is given by the equation $(w-c) \sum_{j=2}^{n} q_{j}^{*}=0$. Solution of this equation is $w^{U}=c$. The incumbent overall profit is $\Pi_{1}^{U}=\left(P\left(Q^{*}\right)-c\right) q_{1}^{*}$. Equation (4) tells us that $\Pi_{1}^{U}>0$. Since $\Pi_{1}^{U}>0$ and $\Pi_{1}^{F B}<0$, it follows from the 
intermediate value theorem that there exist $w \in\left(w^{F B}, w^{U}\right)$ such that $\Pi_{1}(w)=0$.This proves that $w^{O}<w^{U}$. Lemma 3 then implies that $W^{O}<W^{U}$.

Step 3: $\Pi^{M}>\Pi^{U}$ and $W^{M}<W^{U}$

Proof: First we show that $w^{M} \in\left\{w: q_{-1}^{*}(w)=0\right\}$ and $\Pi_{1}^{M}=\left(P\left(Q^{M}\right)-c\right) Q^{M}$ where $Q^{M}$ maximizes $\Pi=(P(Q)-c) Q$. Consider by contradiction any access charge $w$ such that $w \notin\left\{w: q_{-1}^{*}(w)=0\right\}$. The incumbent's profit is $\Pi_{1} \leq(P(Q)-c) Q-\sum_{j=2}^{N} \Pi_{j}$. Lemma 1 implies $\sum_{j=2}^{N} \Pi_{j}>0$, which together with $(P(Q)-c) Q \leq \Pi_{1}^{\mathrm{M}}$ imply that $\Pi_{1}<\Pi_{1}^{\mathrm{M}}$.

Under the second best access charge $w^{U}=c$, itholds that $q_{-1}^{*}>0$. As a consequence $\Pi^{M}>\Pi^{U}$. Lemma 2 further shows that any $w^{M}>w^{U}$. Applying Lemma 3, this proves the second part of the claim. 